\title{
Photodissociation of mono- and di-anionic tin clusters ${ }^{\star}$
}

\author{
Markus Wolframª, Steffi Bandelow, Alexander Jankowski, Stephan König, Gerrit Marx, and Lutz Schweikhard \\ Institute of Physics, University of Greifswald, Greifswald, Germany
}

Received 4 December 2019 / Received in final form 27 March 2020

Published online 25 June 2020

(C) The Author(s) 2020. This article is published with open access at Springerlink.com

\begin{abstract}
Negatively charged tin clusters offer a broad range of decay products, as observed after electroncluster interactions [S. König, M. Wolfram, S. Bandelow, G. Marx, L. Schweikhard, Eur. Phys. J. D 72 , 153 (2018)]. To get further insight into their decay pathways, size and charge-state selected clusters $\mathrm{Sn}_{n}^{-}$ and $\mathrm{Sn}_{n}^{2-}$ were photo-excited at the ClusterTrap setup by $532 \mathrm{~nm} \mathrm{Nd:YAG} \mathrm{laser} \mathrm{pulses.} \mathrm{For} \mathrm{small} \mathrm{mono-}$ anionic precursor clusters containing up to $n \simeq 45$ atoms, $\mathrm{Sn}_{n-7}^{-}$and $\mathrm{Sn}_{n-10}^{-}$are observed as preferred ionic fragments. For bigger clusters a transition to neutral monomer evaporation was found. In the case of di-anionic precursors, preferred decay products are $\mathrm{Sn}_{10}^{-}$and $\mathrm{Sn}_{n-10}^{-}$, indicating fission similar to the case of the group 14 neighbor element lead [S. König, A. Jankowski, G. Marx, L. Schweikhard, M. Wolfram, Phys. Rev. Lett. 120, 163001 (2018)]. Furthermore, doubly charged fragment clusters such as $\mathrm{Sn}_{n-7}^{2-}$ are observed, originating from break-off of neutral heptamers $\mathrm{Sn}_{7}$, a behavior which has not been observed previously for di-anionic clusters.
\end{abstract}

\section{Introduction}

The group 14 elements $(\mathrm{C}, \mathrm{Si}, \mathrm{Ge}, \mathrm{Sn}$, and $\mathrm{Pb})$ show a significant variation in bulk-phase properties, reflecting their different structures and bonding characteristics. In contrast to semiconducting silicon and germanium, bulk tin is metallic under ambient conditions with a tetragonal lattice structure. However, below $286 \mathrm{~K}$ the semiconducting covalently bound Sn (cubic diamond) is the thermodynamically most stable allotrope. The large variation of different structures and bonding characteristics holds true not only for the bulk-phases property, but also for the atomic clusters.

The question, whether ligand-free tin clusters should be classified as metallic or semiconducting, motivated several experimental [1-3] and theoretical [3-5] studies. Singly charged tin cluster anions have been investigated by photoelectron spectroscopy [6-8]. Based on such measurements Wang and coworkers assigned a nonmetal-to-metal transition between $\mathrm{Sn}_{41}^{-}$and $\mathrm{Sn}_{42}^{-}$[9]. In addition, several theoretical investigations focused on the geometric structures of small tin cluster anions [10,11]. The other clusters of the group 14 show similar fragmentation behavior: they break into bigger fragment clusters instead of evaporating monomers [12]. Singly positive charged silicon and germanium clusters break into charged clusters of size $n=6-11$ or break off neutral heptamers and decamers [13-16]. For lead clusters, on the other hand, besides heptamer

* Contribution to the Topical Issue "Atomic Cluster Collisions (2019)", edited by Alexey Verkhovtsev, Pablo de Vera, Nigel J. Mason, Andrey V. Solov'yov.

a e-mail: markus.wolfram@uni-greifswald.de break-off also monomer evaporation was observed [17-19], as known for simple metals [20,21]. Anionic tin clusters are known to break into stable $\mathrm{Sn}_{9}^{-}$and $\mathrm{Sn}_{10}^{-}$and break off neutral heptamers and decamers [22-24].

In the present work, mass-selected negatively charged tin clusters $\operatorname{Sn}_{n}^{-}(n=9-70)$ were photoexcited to further study the dissociation behavior. In addition, the photoexcitation experiments have been extended to mass- and charged-state selected di-anionic tin clusters $\mathrm{Sn}_{n}^{2-}(n=$ 27-70).

\section{Experimental setup and procedure}

The experiments were performed using the ClusterTrap setup, which has already been described in detail [25-27] and followed the procedures applied recently for the investigation of lead clusters [18]. Therefore, only a short overview is given. The experimental steps are illustrated by the set of time-of-flight mass spectra in Figure 1.

Singly negatively charged tin clusters are produced in a laser ablation source [28] and guided into a linear radiofrequency trap (Paul trap), where several ion bunches are accumulated. By use of a quadrupole bender, the ions are transferred from the Paul trap to a Penning Trap with a $12 \mathrm{~T}$ magnet. There, the ion motion is cooled by argon buffer gas (Fig. 1a) and the cluster species of interest, in this example $\mathrm{Sn}_{50}^{-}$, is centered by mass-selective quadrupolar excitation [29] (Fig. 1b). In an optional step, doubly negatively charged clusters are produced with the electron bath technique [30,31] (Fig. 1c), followed by a second selection step (Fig. 1d). 


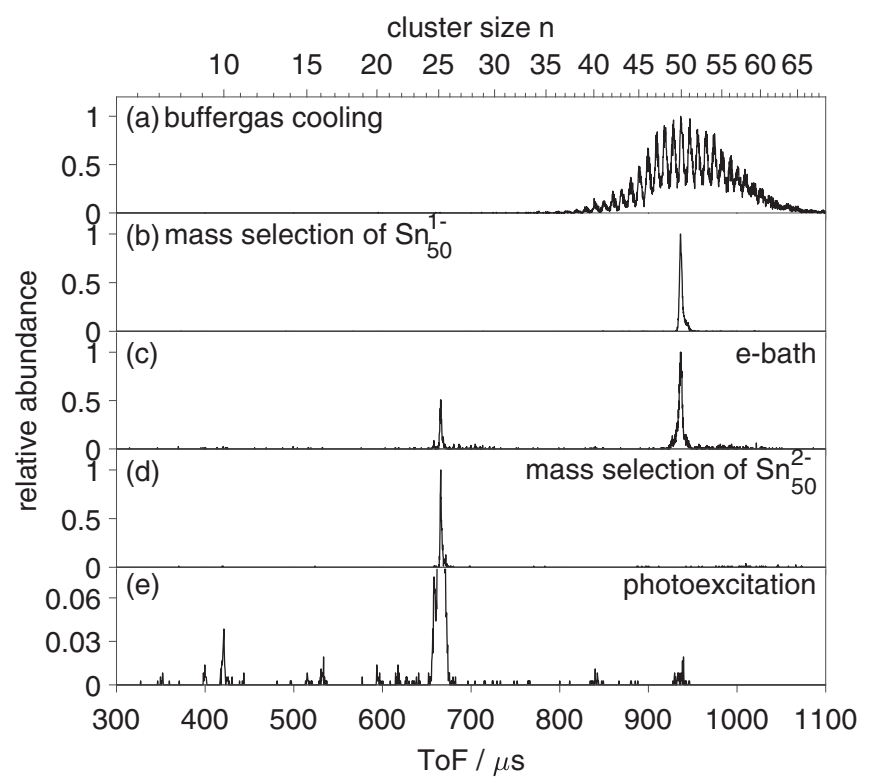

Fig. 1. Top to bottom: time-of-flight (ToF) spectrum after buffer-gas cooling of tin clusters $(40 \lesssim n \lesssim 60)$ in the Penning trap (a), after size selection of $\mathrm{Sn}_{50}^{-}$(b), after electron bath of $\mathrm{Sn}_{50}^{-}$(c), after selection of $\mathrm{Sn}_{50}^{2-}$ (d), and after photoexcitation (e) (for more details see text).

The cluster species of interest is then irradiated with a pulse of a Nd:YaG laser $(532 \mathrm{~nm}, 10 \mathrm{~ns})$. After a delay of $95 \mathrm{~ms}$ the ions (remaining precursors and products) are ejected from the trap and analyzed via time-of-flight mass spectrometry (Fig. 1e).

Photoexcitation was performed for mono-anionic tin clusters of cluster sizes $n=9-70$ and di-anionic tin clusters of cluster sizes $n=27-70$. Typically, a pulse energy of $10 \mathrm{~mJ}$ has been used. For mono- and di-anionic clusters of $n>49$, the pulse energy has been raised to $30 \mathrm{~mJ}$ to increase the product yields. The results are described in the following sections, first for the singly charged and then for the doubly charged anionic clusters.

\section{Photoexcitation of mono-anionic clusters}

Typical cluster abundance spectra are shown in Figure 2 for the example of $\mathrm{Sn}_{36}^{-}$before (Fig. 2a) and after photoexcitation (Fig. 2b). In this case, eight prominent signals are found in addition to the precursor cluster with mass-to-charge-ratios corresponding to tin clusters $\mathrm{Sn}_{n}^{-}$; $n=9,10,15,16,19,22,26,29$. Plausible pathways for these fragments are the sequential break-off starting with neutral heptamers $\mathrm{Sn}_{7}$

$$
\mathrm{Sn}_{36}^{-} \rightarrow \mathrm{Sn}_{29}^{-}+\mathrm{Sn}_{7}
$$

or decamers $\mathrm{Sn}_{10}$

$$
\mathrm{Sn}_{36}^{-} \rightarrow \mathrm{Sn}_{26}^{-}+\mathrm{Sn}_{10}
$$

which have already been reported to be stable neutral clusters [32] and preferred pathways for collision

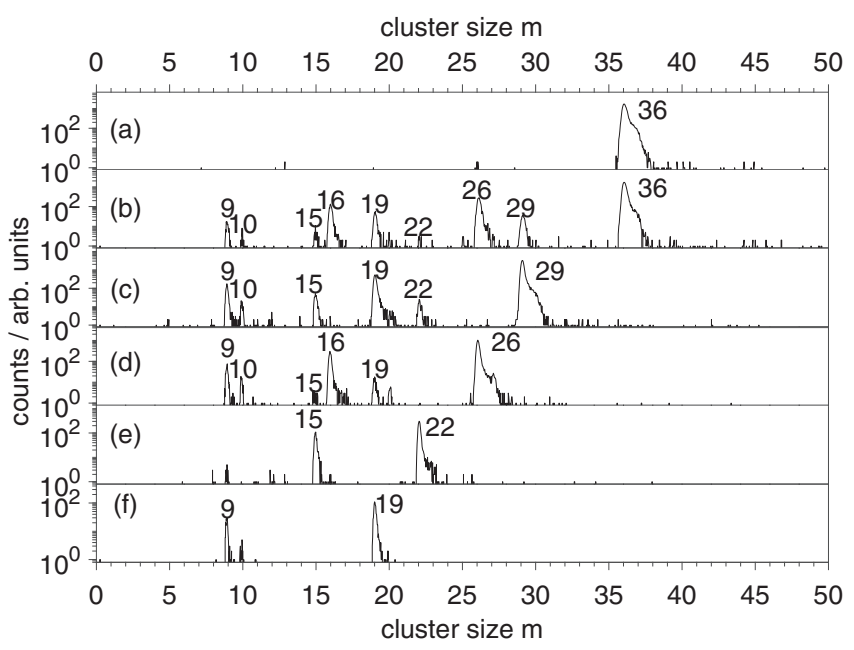

Fig. 2. Spectra of size-selected mono-anionic $\mathrm{Sn}_{36}^{-}$clusters (a) and abundance spectra after photoexcitation of $\mathrm{Sn}_{36}^{-}$(b), $\mathrm{Sn}_{29}^{-}$ (c), $\mathrm{Sn}_{26}^{-}$(d), $\mathrm{Sn}_{22}^{-}$(e) and $\mathrm{Sn}_{19}^{-}$(f) in descending order.

induced dissociation [23] and fragmentation after electron interaction [24].

The following scheme summarizes the decay pathways observed for the case of $\mathrm{Sn}_{36}^{-}$clusters (Figs. 2b-2f) as explained by break-off of neutral hepatmers $\mathrm{Sn}_{7}$ and decamers $\mathrm{Sn}_{10}$. Each line represents a generation of decayproduct clusters, i.e. after the precursor cluster $\mathrm{Sn}_{36}^{-}$on top in the first line, the first generation with $\mathrm{Sn}_{26}^{-}$and $\mathrm{Sn}_{29}^{-}$in the second line, the second generation with $\mathrm{Sn}_{16}^{-}$, $\mathrm{Sn}_{19}^{-}$and $\mathrm{Sn}_{22}^{-}$in the third line, and the third generation, $\mathrm{Sn}_{6}^{-}, \mathrm{Sn}_{9}^{-}, \mathrm{Sn}_{12}^{-}$and $\mathrm{Sn}_{15}^{-}$in the bottom line:

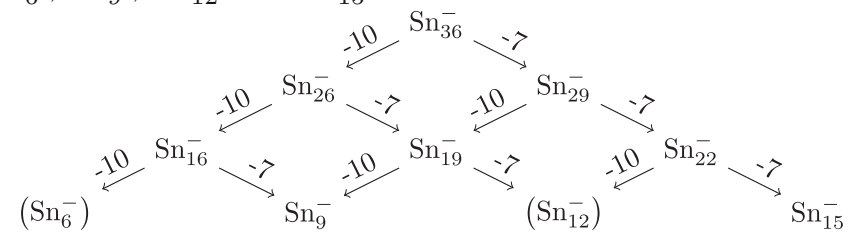
Note that $\mathrm{Sn}_{6}^{-}$and $\mathrm{Sn}_{12}^{-}$are missing in the decay spectra. While the data do not exclude the direct break-off of neutral $\mathrm{Sn}_{17}, \mathrm{Sn}_{20}, \mathrm{Sn}_{21}$ and $\mathrm{Sn}_{27}$ from $\mathrm{Sn}_{36}^{-}$, the set of spectra in Figure 2 suggests sequential break-off of $\mathrm{Sn}_{7}$ and $\mathrm{Sn}_{10}$ fragments.

The decay path behavior changes for larger clusters. As an example, mass spectra of size-selected (top) and photoexcited (bottom) $\mathrm{Sn}_{52}^{-}$clusters are shown in Figure 3. In this case the most dominant fragment is $\mathrm{Sn}_{51}^{-}$, i.e. the monomer-evaporation product, as well as further signals to less extent at the cluster sizes $\mathrm{Sn}_{n}^{-}, n=50,49, \ldots 46$, which suggests sequential monomer evaporation. This behavior is well known for metallic clusters such as gold [33,34], silver [35], copper [36] and aluminum [37]. A signal at $\mathrm{Sn}_{45}^{-}$can still be observed at a higher abundance than the fragments assigned to the sequential monomer evaporation which suggests that the break-off of a neutral heptamer $\mathrm{Sn}_{7}$ remains a significant fragmentation pathway. There is also a small $\mathrm{Sn}_{42}^{-}$signal, possibly due to $\mathrm{Sn}_{10}$ break-off. Further ionic products are observed in the 
cluster size $\mathrm{m}$

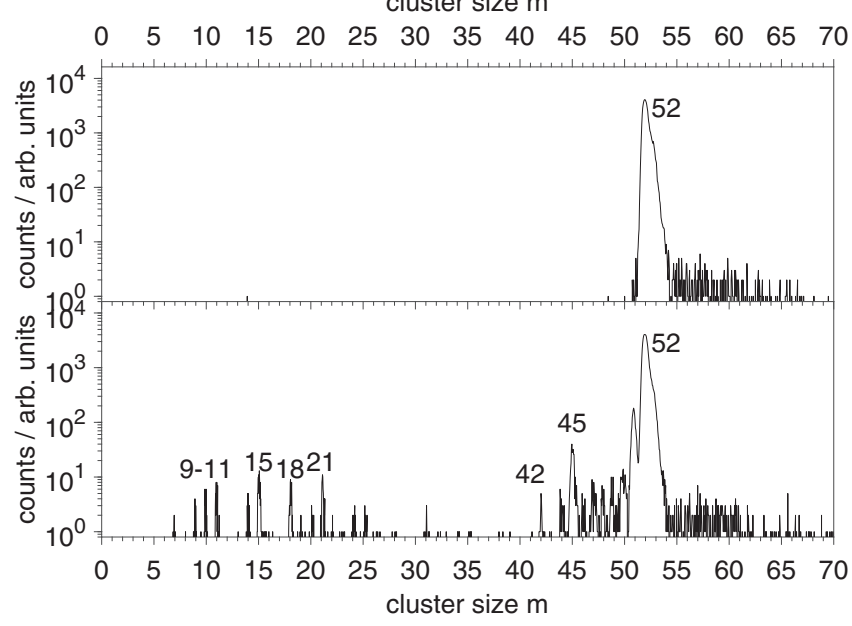

Fig. 3. Spectra of size-selected mono-anionic $\mathrm{Sn}_{52}^{-}$clusters before (top) and after photoexcitation (bottom).

cluster size range of $n=7-31$, in particular $\mathrm{Sn}_{9-11}^{-}, \mathrm{Sn}_{15}^{-}$, $\mathrm{Sn}_{18}^{-}$and $\mathrm{Sn}_{21}^{-} \cdot \mathrm{Sn}_{31}^{-}$as well as $\mathrm{Sn}_{24}^{-}$could arise by sequential decay of neutral heptamers: $\mathrm{Sn}_{52}^{-} \rightarrow \mathrm{Sn}_{45}^{-} \rightarrow \mathrm{Sn}_{38}^{-} \rightarrow$ $\mathrm{Sn}_{31}^{-} \rightarrow \mathrm{Sn}_{24}^{-}$. But $\mathrm{Sn}_{38}^{-}$is missing in the spectrum, maybe due to a higher instability than $\mathrm{Sn}_{45}^{-}$and $\mathrm{Sn}_{31}^{-} \cdot \mathrm{Sn}_{21}^{-}$could be produced by break-off of a neutral decamer from $\mathrm{Sn}_{31}^{-}$: $\mathrm{Sn}_{31} \rightarrow \mathrm{Sn}_{21}^{-}+\mathrm{Sn}_{10}$.

A similar transition in fragmentation behavior as observed between these two examples was already reported for the neighboring transition metal lead [18] and interpreted as a signature of semiconductor-metal transition $[38,39]$. However, in the case of lead the large clusters did not retain the break-off of bigger fragments after this transition.

Photoexcitation experiments have been performed on clusters of the sizes $n=9$ upto 70 with the exception of $\mathrm{Sn}_{12,13}^{-}$, which were not produced in sufficient abundance. An overview of the resulting decay spectra is given in Figure 4: the $x$-axis represents the mass-to-charge ratio $m / z$ of the reaction products (and remaining precursors), the $y$-axis the precursor cluster size. The logarithmic relative abundance is indicated by the gray levels.

The figure can be divided into two different regimes of decay behavior: for clusters smaller than about $\mathrm{Sn}_{45}^{-}$, fragments of sizes $\mathrm{Sn}_{n-k}^{-} ; k=7,10,17,20$ (diagonal lines in Fig. 4) as well as $\mathrm{Sn}_{m}^{-} ; m=10,15$ (vertical lines in Fig. 4) are observed. As described above for the case of the decay of $\mathrm{Sn}_{36}^{-}$, the $\mathrm{Sn}_{n-k}^{-}$can be explained by (sequential) heptamer and/or decamer break-off. In contrast, for clusters bigger than $\mathrm{Sn}_{45}^{-}$, the ionic intensity of product clusters below $m=n-7$ is reduced, while sequential evaporation of neutral monomers dominates.

A closer look at the fragments' abundances is taken in Figure 5. In the top the relative abundance of all fragments with respect to the corresponding precursor is shown as a function of cluster size $n$. The bar plot at the bottom shows the corresponding relative abundances of individual fragment sizes normalized to the sum of all fragments. The plot can be separated into different groups of fragments indicated by differently colored bars: sequential monomer evaporation (black and gray), $\mathrm{Sn}_{7}$ break-off (blue/cyan), $\mathrm{Sn}_{10}$ break-off (yellow/orange), $\mathrm{Sn}_{n-17}^{-}$fragments (green) as combination of both $\mathrm{Sn}_{7}$ and $\mathrm{Sn}_{10}$ as well as further fragments $\mathrm{Sn}_{n-k}^{-}$(magenta), which include larger neutral fragments $k>20$ (or sequential neutral fragment breakoff) and charged clusters such as $\mathrm{Sn}_{9,10,11}^{-}$and $\mathrm{Sn}_{14,15,16}^{-}$.

Both plots of Figure 5 show a shift in fragmentation behavior at the cluster size range of $n \simeq 45-50$, in which the relative abundance of all fragments decreases from $10 \%$ to $40 \%$ to less than $1 \%$, while the relative proportion of monomer evaporation increases from less than $5 \%$ to $70 \%-90 \%$. In the bar plot between $n=$ 14 and 45 a reoccurring pattern is visible, alternating between high relative abundances of fragments from $\mathrm{Sn}_{7}$ and $\mathrm{Sn}_{10}$ break-off. The corresponding abundance maxima can be found at precursor cluster sizes $n$, for which the resulting fragment $\left(\mathrm{Sn}_{n-7}^{-}, \mathrm{Sn}_{n-10}^{-}\right)$are stable cluster sizes $\left(\mathrm{Sn}_{9,10,11}^{-}, \mathrm{Sn}_{14,15,16}^{-}\right.$and $\left.\mathrm{Sn}_{20}^{-}\right)$, i.e. the precursor cluster sizes $n=18,22,27,32$ and 37 show high abundances due to $\mathrm{Sn}_{7}$ break-off in addition to $\mathrm{Sn}_{n-17}^{-}$fragments for precursor sizes $n \geq 27$, while the precursor sizes $n=20,25,30$ and 35 show high abundances due to $\mathrm{Sn}_{10}$ break-off. Further looking at the top plot shows that the same precursor cluster sizes $n=20,25,30$ and 35 mark local maxima for fragment abundance. The particular case of $\mathrm{Sn}_{n-20}^{-}$fragments shows maxima at sizes $n=30,35$ and also 40 and 45.

At those precursor cluster sizes between $n=19$ and $n=$ 45 where the $\mathrm{Sn}_{7}$ break-off does not lead to stable product clusters, the fragmentation processes are dominated by $\mathrm{Sn}_{10}$ break-off. Above the transition at cluster size $n \simeq$ 45 , the $\mathrm{Sn}_{10}$ break-off almost vanishes, while the $\mathrm{Sn}_{n-7}^{-}$ fragments are still occurring at the level of about $10 \%$.

\section{Photoexcitation of di-anionic clusters}

The discussion in the previous section focused on the decay of singly charged $\mathrm{Sn}_{n}^{-}$clusters via monomer evaporation or break-off of larger fragments. Electron detachment has not been considered as the neutral product of this process could not be detected directly. A significant loss of the precursor ions, which would have been the only possible indication of electron detachment, was not observed. However, due to the large statistical fluctuations of the ion intensities, it cannot be excluded as a minor decay pathway. The situation changes, when the precursor charge state is increased. This allows the direct observation of the product clusters after electron detachment (as long as not all excess electrons are emitted [40]). In addition, there is further interest in the photodissociation of doubly charged anionic precursors as they may well break up into two singly charged fragments, a process recently observed in the case of lead clusters [19] and called fission in the following.

As an example, Figure 6 shows abundance spectra before (top) and after photoexcitation (bottom) of $\mathrm{Sn}_{37}^{2-}$. For this cluster size, neither electron detachment to $\mathrm{Sn}_{37}^{-}$ 


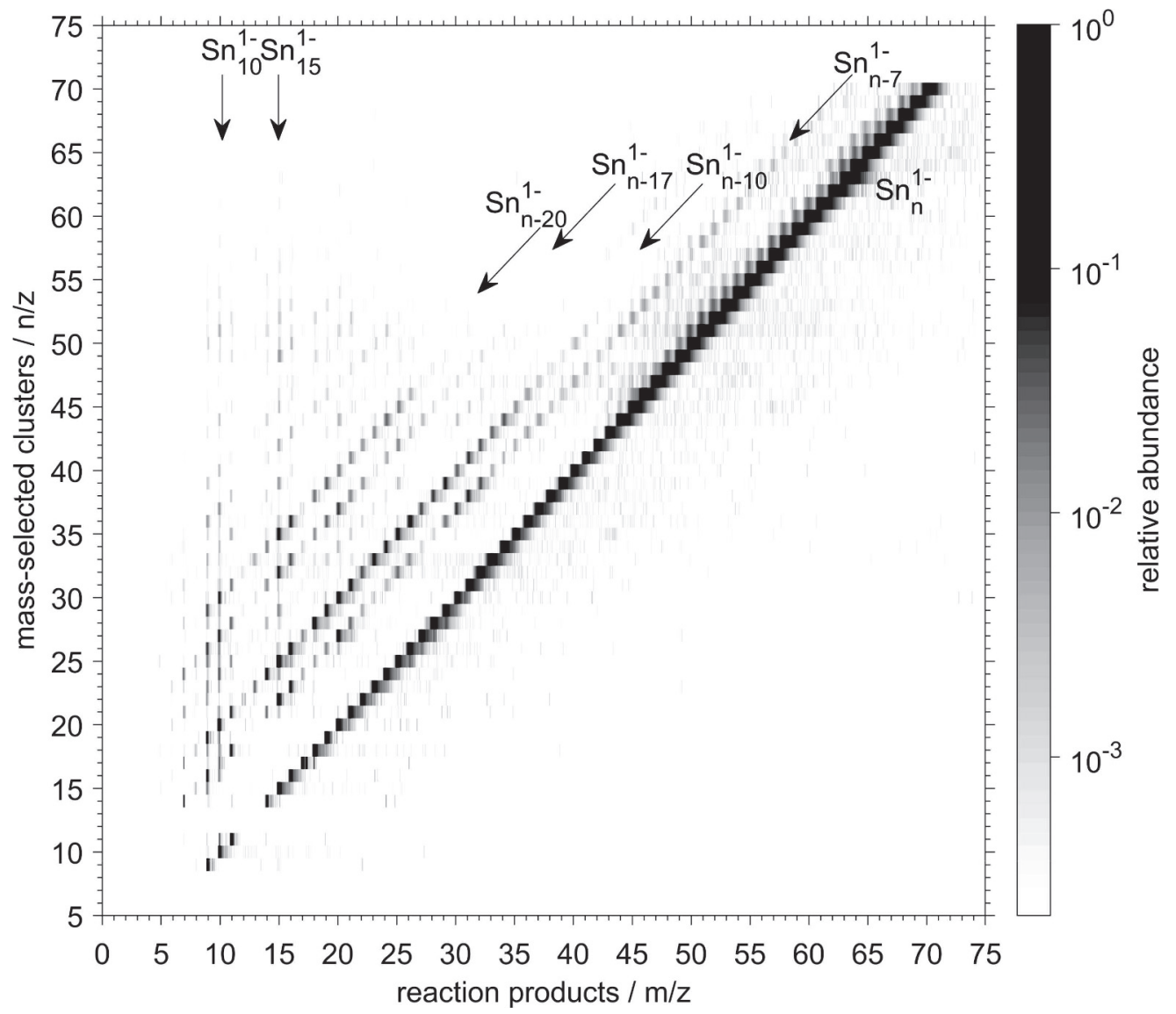

Fig. 4. Overview of $\mathrm{Sn}_{n}^{-}$decay spectra (for details see text).

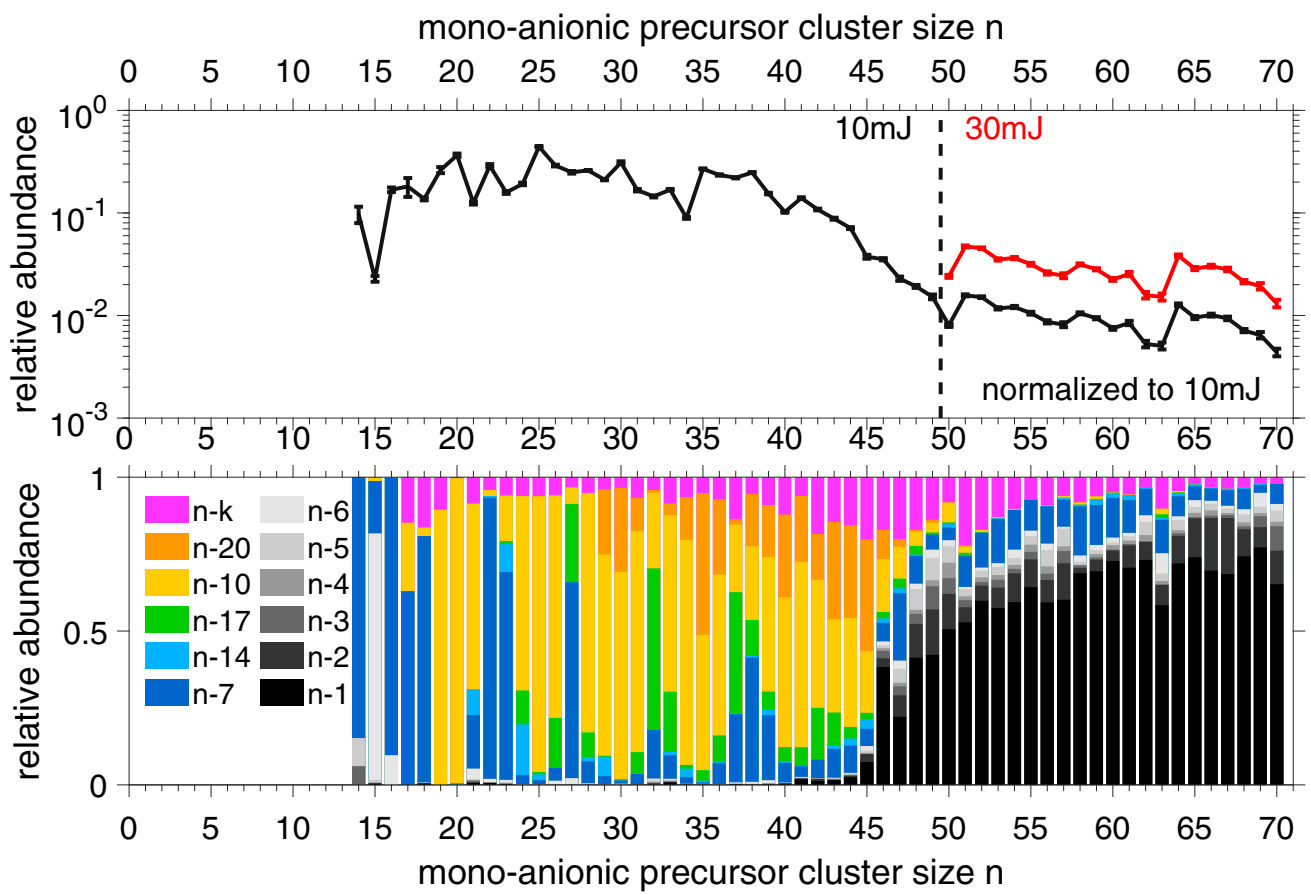

Fig. 5. Top: fragment abundance over precursor cluster size $n$ after photoexcitation of $\operatorname{Sn}_{n}^{-}$. Bottom: sum of most abundant fragments normalized to fragment abundance as a function of precursor cluster size $n$. 


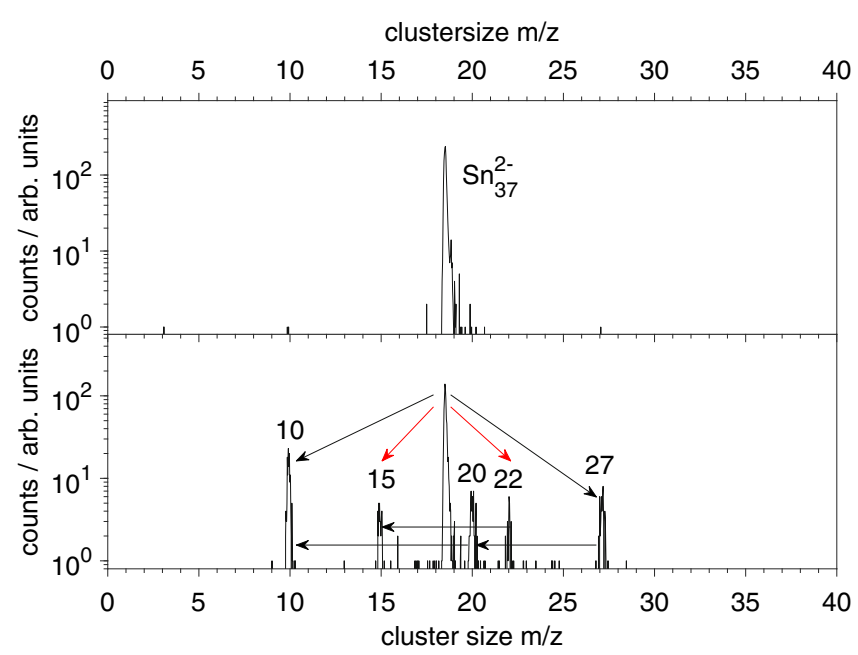

Fig. 6. Spectra of mass-selected $\mathrm{Sn}_{37}^{2-}$ clusters before (top) and after photo excitation (bottom).

nor significant (sequential) neutral monomer evaporation to $\mathrm{Sn}_{36}^{2-}\left(\mathrm{Sn}_{35}^{2-}, \mathrm{Sn}_{34}^{2-}, \ldots\right)$ is observed. Instead, prominent signals show up at $n / z=10,15,20,22$ and 27, which can be interpreted as two fission processes, namely

$$
\mathrm{Sn}_{37}^{2-} \rightarrow \mathrm{Sn}_{15}^{-}+\mathrm{Sn}_{22}^{-}
$$

and

$$
\mathrm{Sn}_{37}^{2-} \rightarrow \mathrm{Sn}_{10}^{-}+\mathrm{Sn}_{27}^{-}
$$

as well as a subsequent neutral heptamer break-off,

$$
\mathrm{Sn}_{27}^{-} \rightarrow \mathrm{Sn}_{20}^{-}+\mathrm{Sn}_{7}
$$

As described in the previous section, singly charged clusters of size $n=10$ and $n=15$ are preferred reaction products of photoexcited mono-anionic tin clusters. The present results again hint at an increased stability of these clusters.

The signal of $\mathrm{Sn}_{10}^{-}$is more intense than the one of the associated fission product $\mathrm{Sn}_{27}^{-}$, which is still excited and can undergo (several) subsequent decay steps. For example it can undergo another decay to $\mathrm{Sn}_{20}^{-}$by neutral heptamer evaporation. Even the combined abundance of the products $\mathrm{Sn}_{20}^{-}$and $\mathrm{Sn}_{27}^{-}$is only $3 / 5$ of that of $\mathrm{Sn}_{10}^{-}$. As known from Section 3 , there can be sequential decays to $\mathrm{Sn}_{10}^{-}$, namely the decay of $\mathrm{Sn}_{27}^{-}$by sequential break-off of a heptamer and a decamer (in whatever order, with a summed probability of 0.25 according to Fig. 5), as well as $\mathrm{Sn}_{20}^{-} \rightarrow \mathrm{Sn}_{10}^{-}+\mathrm{Sn}_{10}$, which is the only observed decay channel for $\mathrm{Sn}_{20}^{-}$.

The signal intensities of the fission products $n=15$ and 22 are closer than in the case of $n=10$ and 27. However, that of $n=22$ is still ca. $1 / 3$ smaller than that of $n=15$. Again, a subsequent decay of the less stable product, namely $\mathrm{Sn}_{22}^{-}$, seems likely. Following the results of the previous section, a decay of $\mathrm{Sn}_{22}^{-}$would occur by neutral heptamer evaporation with a likelihood of 0.9 , leading to the observed increased production of the product $\mathrm{Sn}_{15}^{-}$.
In principle, the signal of $\mathrm{Sn}_{15}^{-}$could also be superimposed by di-anionic clusters $\mathrm{Sn}_{30}^{2-}$, produced by neutral heptamer evaporation from the precursor cluster itself. However, this process seems to emerge only at bigger precursor cluster sizes, as discussed in the following.

Figure 7 shows the photodissociation spectra of dianionic tin clusters in analogy to the spectra of monoanions in Figure 4. Again, two size regimes with different decay behavior can be identified. Clusters up to size $n \sim 50$ prefer to decay by fission processes, e.g. into $\mathrm{Sn}_{10}^{-}$and $\mathrm{Sn}_{n-10}^{-}$as discussed above. Additionally, intense signals at fragment cluster sizes as $\mathrm{Sn}_{n-17}^{-}$and $\mathrm{Sn}_{n-20}^{-}$ are observed, produced by subsequent break-off of neutral subunits. This leads to a multitude of different fragment lines in the lower part of the plot.

For cluster sizes above $n \sim 50$ the decay behavior changes. The relative abundance of the detected fragments decreases to only a few percent as observed for monoanions before. In addition, mono-anionic $\mathrm{Sn}_{n}^{-}$clusters appear in the spectra, i.e. electron detachment is observed. This shift of pattern occurs in the same size-regime as the change to monomer-evaporation observed for excited mono-anionic clusters and may be a further indication of the nonmetal-to-metal-transition. Some of the larger clusters that undergo electron detachment, $\mathrm{Sn}_{n}^{2-} \rightarrow \mathrm{Sn}_{n}^{-}$, have further excitation energy and, thus, subsequently evaporate neutral monomers or decay by heptamer break-off as known from the previous section.

In Figure 8 the spectra before and after photoexcitation of $\mathrm{Sn}_{62}^{2-}$ clusters are presented. Here the break-off of neutral heptamers takes place. This is a new decay and a specific pathway feature characteristic for tin clusters, i.e. not observed in the case of the neighboring element lead of the same group in the periodic table of elements. Figure 9 shows the photoexcitation spectra of $\mathrm{Sn}_{45}^{2-}$, with both break-off of neutral decamers and heptamers, as well as the decamer fission process $\mathrm{Sn}_{45}^{2-} \rightarrow \mathrm{Sn}_{35}^{-}+\mathrm{Sn}_{10}^{-}$in analogy to the case of $\mathrm{Sn}_{37}^{2-}$ (Fig. 6).

Figure 10 shows, in analogy to Figure 5, in the top the intensity of fragment signals normalized to the sum of all clusters signals (i.e. including the precursor) for each dianionic precursor cluster size. Again, the fragment intensity is decreasing with increasing cluster size. The drops at the precursor sizes $n=30$ and 40 are probably artifacts: For these di-anionic clusters symmetric fission

$$
\begin{aligned}
& \mathrm{Sn}_{30}^{2-} \rightarrow \mathrm{Sn}_{15}^{-}+\mathrm{Sn}_{15}^{-} \\
& \mathrm{Sn}_{40}^{2-} \rightarrow \mathrm{Sn}_{20}^{-}+\mathrm{Sn}_{20}^{-}
\end{aligned}
$$

leads to preferred product clusters. As fragments and precursors have the same values of cluster size over charge state, they cannot be distinguished and these fission processes remain undetected.

The bottom of Figure 10 shows the precursor ranges of the most abundant decay pathways (listed on the left). Cluster sizes where only a given decay process is observed are indicated by blue lines, while precursors that show sequential decay are marked by red lines. In particular, for the large cluster-size range from $n=27-51$, decamer-fission reaction products perform further decays. 


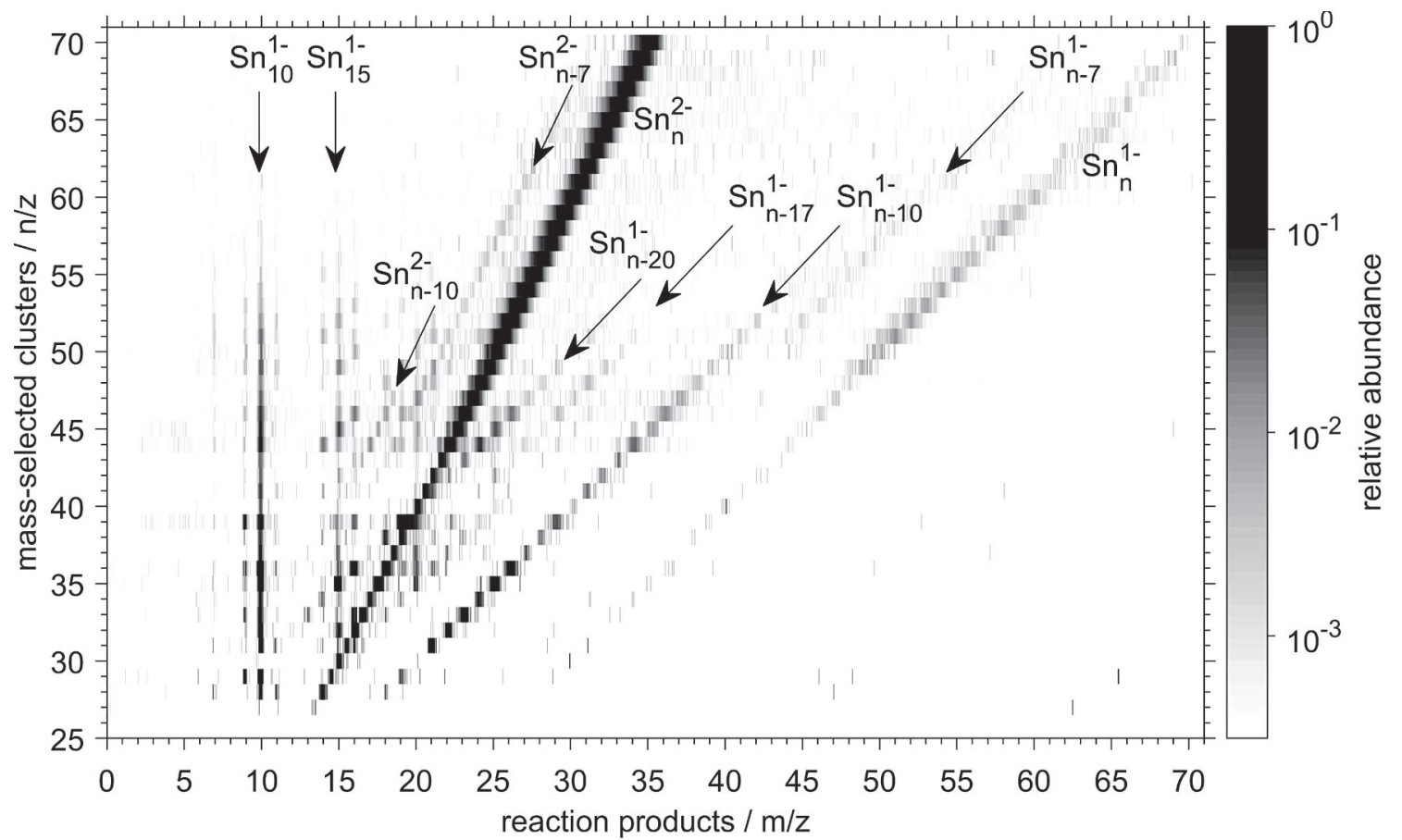

Fig. 7. Overview of $\mathrm{Sn}_{n}^{2-}$ decay spectra (for details see text).

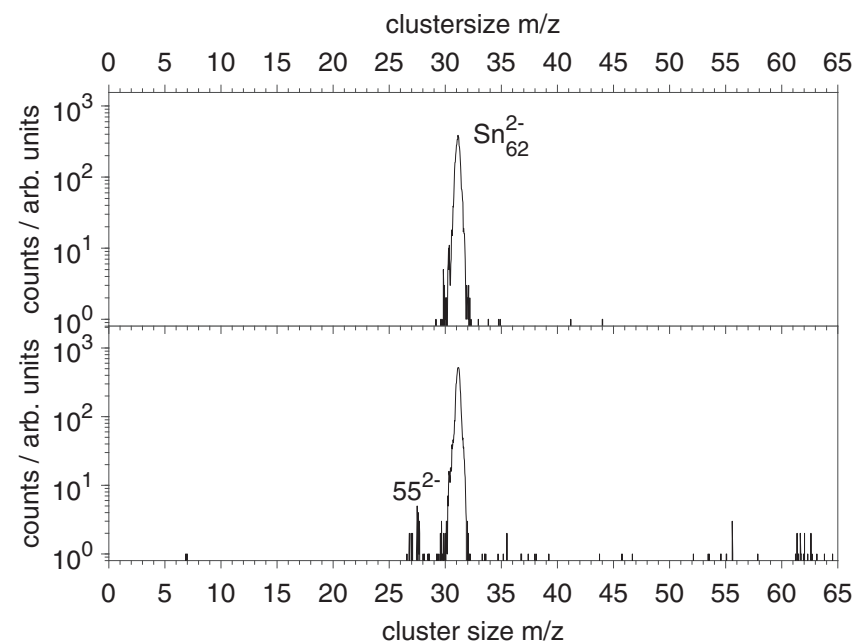

Fig. 8. Spectra of mass-selected $\mathrm{Sn}_{62}^{2-}$ clusters before (top) and after photoexcitation (bottom).

Only $\mathrm{Sn}_{30}^{2-}$ is an exception, which seems to fission into $\mathrm{Sn}_{15}^{-}+\mathrm{Sn}_{n-15}^{-}$. However, as discussed above, symmetric fission processes were not accessible in the present measurements.

Overall, the figure shows again the shift from a size range of mainly fission processes to a second range, where electron detachment and monomer evaporation dominates, reminiscent of the same behavior in the case of lead clusters [18]. However, the lack of electron detachment for cluster sizes near the di-anion appearance size is surprising, since for other metal clusters such as gold electron emission is the predominant decay process [40].

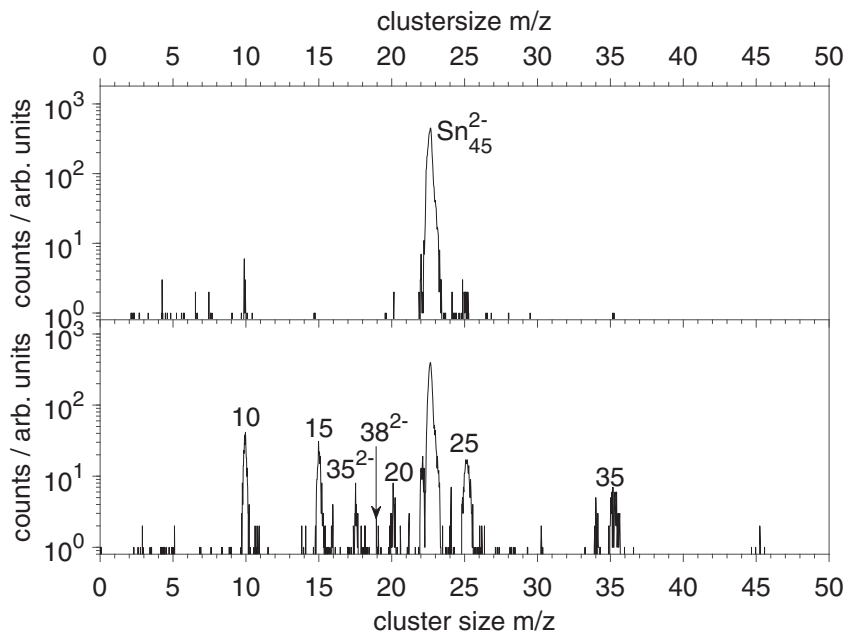

Fig. 9. Spectra of mass-selected $\mathrm{Sn}_{45}^{2-}$ clusters before (top) and after photoexcitation (bottom).

\section{Summary and outlook}

Size selected mono-anionic $(n=9-70)$ and di-anionic $(n=27-70)$ tin clusters were photoexcited to study their dissociation behavior. For both mono-anionic and di-anionic clusters there are two size regimes. For smaller mono-anionic tin clusters up to a size of $n \simeq 45$ breakoff of neutral $\mathrm{Sn}_{7}$ and $\mathrm{Sn}_{10}$ is observed. For the bigger ones monomer evaporation becomes the dominant decay process. This confirms the suggestion of a nonmetal-tometal-transition [9] in analogy to the lead-cluster behavior discussed in [18]. 

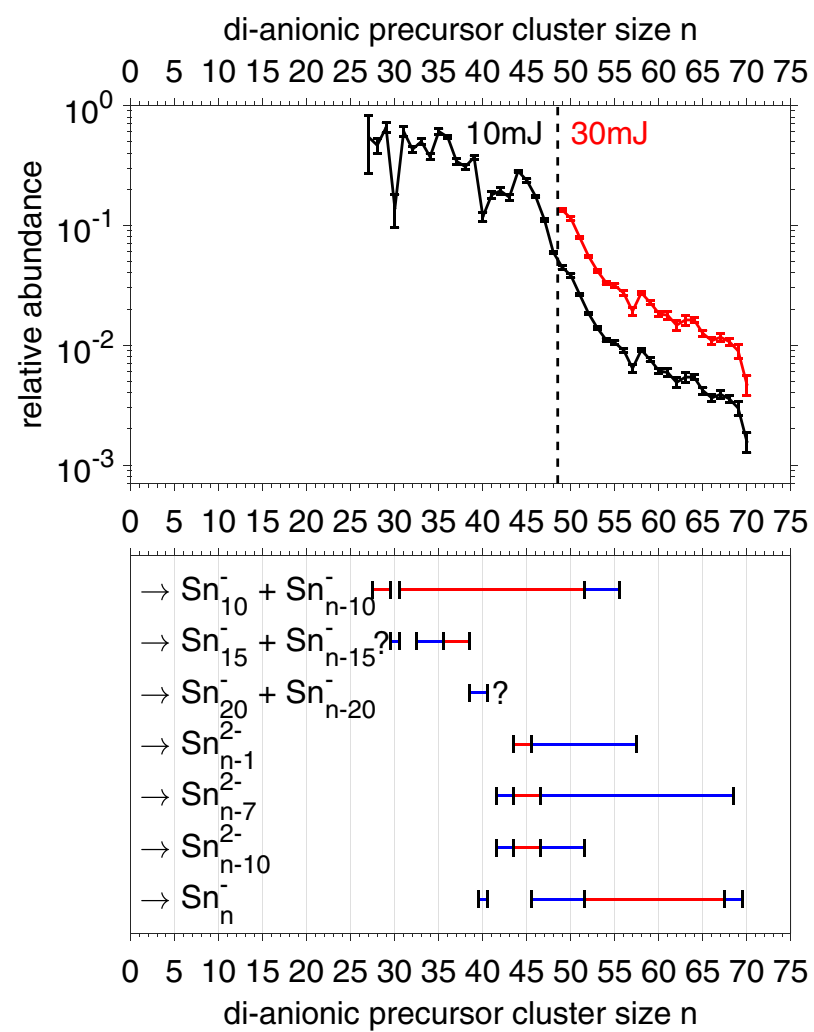

Fig. 10. Top: relative abundance of all fragments as a function of cluster size of the dianionic precursors $\mathrm{Sn}_{27-70}^{2-}$. For the cluster sizes $n \geq 49$ the laser-pulse energy was increased to $30 \mathrm{~mJ}$ (red points) and the results were divided by 3 for normalization to $10 \mathrm{~mJ}$ (black points). Bottom: overview of the main decay pathways (indicated on the left). The cluster-size ranges of these decay pathways are shown by horizontal lines, where red includes sequential decays while blue indicates the occurrence of only single-step processes (for more details see text).

For small di-anionic tin clusters $n \leq 50$ fission into $\mathrm{Sn}_{10}^{-}$ and $\mathrm{Sn}_{n-10}^{-}$is the predominant process. But also fission into $\mathrm{Sn}_{15}^{-}$and $\mathrm{Sn}_{n-15}^{-}$was observed. The bigger clusters decay by electron detachment as known for other metal clusters. But also the break-off of neutral sub-units such as $\mathrm{Sn}_{7}$ and $\mathrm{Sn}_{10}$ becomes a decay channel. This feature had not been observed previously for di-anionic metal clusters.

For a better disentanglement of the decay channels, time-resolved measurements at appropriate photon energies are planned, as already reported for lead clusters [41].

Open access funding provided by Projekt DEAL.

\section{Author contribution statement}

M.W. and A.J. performed the measurements and analyzed the data. L.S. and G.M. planned and supervised the research. M.W., L.S., A.J., S.K. and S.B. wrote the manuscript. All authors helped to perform the experiment, contributed to the discussion and commented on the manuscript at all stages.
Publisher's Note The EPJ Publishers remain neutral with regard to jurisdictional claims in published maps and institutional affiliations.

Open Access This is an open access article distributed under the terms of the Creative Commons Attribution License (https://creativecommons.org/licenses/by/4.0/), which permits unrestricted use, distribution, and reproduction in any medium, provided the original work is properly cited.

\section{References}

1. R.E. Honig, J. Chem. Phys. 21, 573 (1953)

2. A.A. Shvartsburg, M.F. Jarrold, Phys. Rev. A 60, 1235 (1999)

3. A.A. Shvartsburg, M.F. Jarrold, Phys. Rev. Lett. 85, 2530 (2000)

4. C. Majumder, V. Kumar, H. Mizuseki, Y. Kawazoe, Phys. Rev. B 64 (2001)

5. K. Joshi, D.G. Kanhere, S.A. Blundell, Phys. Rev. B 67 (2003)

6. G. Ganteför, M. Gausa, K.H. Meiwes-Broer, H.O. Lutz, Z. Phys. D: At. Mol. Clusters 12, 405 (1989)

7. V.D. Moravec, S.A. Klopcic, C.C. Jarrold, J. Chem. Phys. 110, 5079 (1999)

8. Y. Negishi, H. Kawamata, A. Nakajima, K. Kaya, J. Electron Spectrosc. Relat. Phenom. 106, 117 (2000)

9. L.F. Cui, L.M. Wang, L.S. Wang, J. Chem. Phys. 126, 064505 (2007)

10. P. Jackson, I. Dance, K. Fisher, G. Willett, G. Gadd, Int. J. Mass Spectrom. Ion Process. 157-158, 329 (1996)

11. C. Zhao, K. Balasubramanian, J. Chem. Phys. 115, 3121 (2001)

12. S. Yoshida, K. Fuke, J. Chem. Phys. 111, 3880 (1999)

13. J.M. Hunter, J.L. Fye, M.F. Jarrold, J.E. Bower, Phys. Rev. Lett. 73, 2063 (1994)

14. M.F. Jarrold, J.E. Bower, J. Phys. Chem. 92, 5702 (1988)

15. J.R. Heath, Y. Liu, S.C. O'Brien, Q.L. Zhang, R.F. Curl, F.K. Tittel, R.E. Smalley, J. Chem. Phys. 83, 5520 (1985)

16. P. Ferrari, E. Janssens, P. Lievens, K. Hansen, J. Chem. Phys. 143, 224313 (2015)

17. R. Kelting, R. Otterstätter, P. Weis, N. Drebov, R. Ahlrichs, M.M. Kappes, J. Chem. Phys. 134, 024311 (2011)

18. S. König, F. Martinez, L. Schweikhard, M. Wolfram, J. Phys. Chem. C 121, 10858 (2017)

19. S. König, A. Jankowski, G. Marx, L. Schweikhard, M. Wolfram, Phys. Rev. Lett. 120, 163001 (2018)

20. P.J. Brucat, L.S. Zheng, C.L. Pettiette, S. Yang, R.E. Smalley, J. Chem. Phys. 84, 3078 (1986)

21. M. Vogel, A. Herlert, L. Schweikhard, J. Am. Soc. Mass. Spectrom. 14, 614 (2003)

22. E. Oger, R. Kelting, P. Weis, A. Lechtken, D. Schooss, N.R.M. Crawford, R. Ahlrichs, M.M. Kappes, J. Chem. Phys. 130, 124305 (2009)

23. A. Wiesel, N. Drebov, T. Rapps, R. Ahlrichs, U. Schwarz, R. Kelting, P. Weis, M.M. Kappes, D. Schooss, Phys. Chem. Chem. Phys. 14, 234 (2012)

24. S. König, M. Wolfram, S. Bandelow, G. Marx, L. Schweikhard, Eur. Phys. J. D 72, 153 (2018)

25. L. Schweikhard, S. Krückeberg, K. Lützenkirchen, C. Walther, Eur. Phys. J. D 9, 15 (1999) 
26. F. Martinez, G. Marx, L. Schweikhard, A. Vass, F. Ziegler, Eur. Phys. J. D 63, 255 (2011)

27. F. Martinez, S. Bandelow, C. Breitenfeldt, G. Marx, L. Schweikhard, A. Vass, F. Wienholtz, Int. J. Mass Spectrom. 365-366, 266 (2014)

28. H. Weidele, U. Frenzel, T. Leisner, D. Kreisle, Z. Phys. D: At. Mol. Clusters 20, 411 (1991)

29. G. Savard, S. Becker, G. Bollen, H.J. Kluge, R. Moore, T. Otto, L. Schweikhard, H. Stolzenberg, U. Wiess, Phys. Lett. A 158, 247 (1991)

30. A. Herlert, S. Krückeberg, L. Schweikhard, M. Vogel, C. Walther, Phys. Scr. T80, 200 (1999)

31. L. Schweikhard, A. Herlert, G. Marx, AIP Conf. Proc. 692, 203 (2003)

32. A. Lechtken, N. Drebov, R. Ahlrichs, M.M. Kappes, D. Schooss, J. Chem. Phys. 132, 211102 (2010)

33. S. Becker, G. Dietrich, H.U. Hasse, N. Klisch, H.J. Kluge, D. Kreisle, S. Krückeberg, M. Lindinger, K. Lützenkirchen, L. Schweikhard et al., Z. Phys. D: At. Mol. Clusters 30, 341 (1994)
34. V.A. Spasov, Y. Shi, K.M. Ervin, Chem. Phys. 262, 75 (2000)

35. S. Krückeberg, G. Dietrich, K. Lützenkirchen, L. Schweikhard, C. Walther, J. Ziegler, Int. J. Mass Spectrom. Ion Process. 155, 141 (1996)

36. S. Krückeberg, L. Schweikhard, J. Ziegler, G. Dietrich, K. Lützenkirchen, C. Walther, J. Chem. Phys. 114, 2955 (2001)

37. M.F. Jarrold, J.E. Bower, J.S. Kraus, J. Chem. Phys. 86, 3876 (1987)

38. B. Waldschmidt, M. Turra, R. Schäfer, Z. Phys. Chem. 221, 1569 (2007)

39. J. Bahn, P. Oelßner, M. Köther, C. Braun, V. Senz, S. Palutke, M. Martins, E. Rühl, G. Ganteför, T. Möller et al., New J. Phys. 14, 075008 (2012)

40. A. Herlert, L. Schweikhard, New J. Phys. 14, 055015 (2012)

41. M. Wolfram, S. König, S. Bandelow, P. Fischer, A. Jankowski, G. Marx, L. Schweikhard, J. Phys. B: At., Mol. Opt. Phys. 51, 044005 (2018) 